\title{
MicroRNA-577 inhibits the migration and invasion of hepatocellular carcinoma cells by targeting homeobox A1
}

\author{
SHAOSHAN HAN, ZHIKUI LIU, YUFENG WANG, LIANG WANG, BOWEN YAO, \\ CHENG GUO, TAO SONG, KANGSHENG TU and QINGGUANG LIU \\ Department of Hepatobiliary Surgery, The First Affiliated Hospital of Xi'an Jiaotong University, \\ Xi'an, Shaanxi 710061, P.R. China
}

Received October 16, 2017; Accepted April 12, 2018

DOI: $10.3892 /$ or.2018.6388

\begin{abstract}
Research has confirmed that abnormally expressed miRNAs are involved in the occurrence and development of hepatocellular carcinoma (HCC). In the present study, we confirmed that miR-577 expression both in HCC tissues and cell lines was markedly downregulated. Clinically, downregulated expression of miR-577 is notably related to malignant clinicopathological features, such as venous invasion and advanced TNM stage. Additionally, miR-577 may act as a valuable tumor marker to predict the prognosis of HCC patients. Through knockdown and overexpression of miR-577, miR-577 was identified as an inhibitor of cell metastatic ability and EMT progress in HCC. Furthermore, miR-577 was able to directly bind to the 3'-UTR of homeobox A1 (HOXA1) to regulate the expression of HOXA1. In addition, there existed a negative correlation between the expression of miR-577 and HOXA1 in HCC specimens. Rescue experiments revealed that the influence of miR-577 on the migration, invasion and EMT of HCC cells was reversed by HOXA1. Taken together, our findings demonstrated that miR-577 functions as an anti-oncogene to suppress the migration, invasion and EMT of HCC cells through direct interaction with HOXA1. miR-577 may act as a valuable target for the molecular-targeted therapy of HCC.
\end{abstract}

Correspondence to: Professor Qingguang Liu, Department of Hepatobiliary Surgery, The First Affiliated Hospital of Xi'an Jiaotong University, 277 Yanta West Road, Xi'an, Shaanxi 710061, P.R. China

E-mail: liuqingguang2017@126.com

Abbreviations: HCC, hepatocellular carcinoma; EMT, epithelialmesenchymal transition; HOXA1, homeobox A1; qPCR, quantitative real-time polymerase chain reaction; UTR, untranslated region

Key words: hepatocellular carcinoma, miR-577, HOXA1, EMT, metastasis

\section{Introduction}

As one of the most common malignant tumors, hepatocellular carcinoma (HCC) has been identified as the third primary cause of tumor-induced death in the word (1). Despite great advancements in the diagnosis and treatment of $\mathrm{HCC}$, the rates of distant metastasis and local recurrence after surgical resection remain high resulting in a poor long-term patient prognosis (2-4). Thus, it is extremely critical to uncover the potential mechanisms underlying HCC progression.

MicroRNAs (miRNAs), a type of endogenous, small and non-coding RNA, are involved in tumor initiation, development and progression via binding with the 3 '-untranslated region (UTR) of target genes, which results in the translational inhibition or degradation of the target mRNAs $(5,6)$. Accumulating data have revealed that aberrant miRNAs are involved in HCC initiation, development and progression, which could represent potential diagnostic, therapeutic and prognostic markers (7). Recent studies have demonstrated that miR-577 is dysregulated in cancers $(8,9)$. miR-577 was found to modulate the Wnt signaling pathway to inhibit glioblastoma tumor growth (10). Yu et al reported that, in gastric cancer, E2F transcription factor 3 works as a direct downstream target of miR-577 (11). Moreover, in colorectal cancer, miR-577 suppressed tumor growth and enhanced chemosensitivity (12). In addition, in research concerning pediatric diabetes, miR-577 was identified as an inhibitor to pancreatic $\beta$-cell function and survival, which targeted fibroblast growth factor 21 (13). These studies suggest that miR-577 is a cancer-related gene. However, the expression and the specific mechanism of miR-577 in HCC remain to be uncovered.

Epithelial-to-mesenchymal transition (EMT) has been confirmed to be critical in tumor metastasis, including HCC (14-16). EMT results in decreased expression of epithelial marker (E-cadherin), while the expression of mesenchymal markers (N-cadherin and vimentin) are enhanced. EMT enhances the migratory and invasive properties of HCC cells, thereby contributing to HCC metastasis (17). However, in HCC, whether miR-577 regulates the process of EMT in tumor cells has been rarely investigated. 
Here, we found that decreased expression of miR-577 was closely related to poor clinicopathological features and HCC patient survival. Functionally, miR-577 suppressed the migration and invasion of HCC cells by directly targeting HOXA1. Additionally, miR-577 inhibited the process of EMT in HCC cells. In conclusion, these findings demonstrated that miR-577 suppressed HCC cell migration, invasion and EMT, and miR-577 may act as a possible valuable target for molecular-targeted therapy of HCC.

\section{Materials and methods}

Tissue samples. HCC tissues and adjacent non-tumor tissues were obtained from patients diagnosed with HCC at the Department of Hepatobiliary Surgery, The First Affiliated Hospital of Xi'an Jiaotong University (Xi'an, China) from January 2007 to December 2009. The HCC patients did not receive any adjuvant therapy before surgery, such as chemotherapy or radiotherapy. The fresh tissues were stored in liquid nitrogen. All of the patients provided written informed consent. Xi'an Jiaotong University Ethics Committee approved the research on the basis of the Declaration of Helsinki.

Cell culture and transfection. The normal hepatic cell line (LO2) and five human HCC cell lines (Huh7, MHCC-97L, MHCC-97H, SMMC-7721 sand Hep3B) were obtained from the Chinese Academy of Sciences (Shanghai, China). Complete Dulbecco's modified Eagle's medium (DMEM) (Invitrogen; Thermo Fisher Scientific, Inc., Waltham, MA, USA) with $1 \%$ penicillin-streptomycin (Thermo Fisher Scientific, Inc.) and 10\% FBS (Gibco; Thermo Fisher Scientific, Inc.) was applied to culture the cells. Cells were then placed in a humidified atmosphere $\left(37^{\circ} \mathrm{C}, 5 \% \mathrm{CO}_{2}\right)$.

Lipofectamine 2000 reagent (Invitrogen Life Technologies; Thermo Fisher Scientific, Inc.) was applied to conduct cell transfection on the basis of the product specification at a final concentration of 100 pmol according to previous studies $(9,10)$. The mimic control (miR-control; CmiR0001-MR04), miR-577 mimics (miR-577, HmiR0074-MR04), inhibitor control (anti-miR-NC; CmiR-AN0001-AM02) and miR-577 inhibitors (anti-miR-577; HmiR-AN0678-AM02) were purchased from Genecopoeia (Guangzhou, China). HOXA1 clones and HOXA1 siRNAs were obtained from Sangon Biotech Co., Ltd. (Shanghai, China). The cells were transfected with the siRNAs and clones above using Lipofectamine 2000 according to the manufacturer's instructions. After $48 \mathrm{~h}$ of transfection, cells were used for the following experiments.

Quantitative real-time PCR ( $q P C R)$. TRIzol (Thermo Fisher Scientific, Inc.) was employed to extract total RNA from the tissues and cells according to the product manual. Then, TIANScript RT Kit (Tiangen Biotech, Beijing, China) was used to synthesize cDNAs. TaqMan Human MiRNA Assay Kit (Genecopoeia, Guangzhou, China) and the SYBR Premix Ex Taq ${ }^{\mathrm{TM}}$ Kit (Takara Bio Inc.,, Shiga, Japan) were used to conduct PCR amplifications for miR-577 and HOXA1 mRNA. The detection was performed in the ABI 7300 system (Applied Biosystems, Foster City, CA, USA). snRNA U6 qPCR Primer (HmiRQP9001), hsa-miR-577 primer (HmiRQP0678), GAPDH (HQP006940) and HOXA1 primer (HQP008966) were purchased from Genecopoeia (Guangzhou, China).

Western blot analysis. Proteins were isolated with RIPA buffer and then separated on 10\% SDS-PAGE gels. After proteins were transferred to PVDF membranes, the membranes were blocked using 5\% non-fat milk/TBST (Tris-buffered saline Tween-20). Subsequently, the primary antibodies rabbit anti-HOXA1 (1:1,000; cat. no. ab37563; Abcam, Cambridge, UK), mouse anti-E-cadherin (cat. no. 14472; Cell Signaling Technology, Inc. Danvers, MA, USA), rabbit anti-N-cadherin (cat. no. 13116; Cell Signaling Technology, Inc.), rabbit anti-vimentin (cat. no. 5741; Cell Signaling Technology, Inc.) were used to incubate the membranes at $4^{\circ} \mathrm{C}$ overnight. Secondary antibodies (anti-rabbit cat. no. 7074 and anti-mouse cat. no. 7076; Cell Signaling Technology, Inc.) were employed and the ECL reagent (Beyotime Institute of Biotechnology, Haimen, China) was applied for detection.

Luciferase reporter assay. The bioinformation public database TargetScan and miRanda was used. Cells were seeded in triplicate in a 24-well plate and pGL3-HOXA1 was co-transfected into HCC cells with the TK-Renilla plasmid as control signals using Lipofectamine 2000. Moreover, vectors with the wild-type HOXA1 3'-UTR or mutant HOXA1 3'-UTR constructed by Sangon Biotech (Shanghai, China) and relevant mir-577 or anti-miR-577 vectors were co-transfected into HCC cells. After $48 \mathrm{~h}$, the luciferase activity was measured by a Dual-Luciferase Reporter Assay system (E1910; Promega, Madison, WI, USA). Three independent experiments were performed and the data are presented as the mean $\pm \mathrm{SD}$.

MTT assays. Cell viability was detected by the 3-(4,5-dimethyl thiazol-2-yl)2,5-diphenyltetrazolium bromide (MTT; Sigma-Aldrich; Merck KGaA, Darmstadt, Germany) assay. Detailed protocol of the experiment was described in previous studies $(18,19)$.

Transwell assays. Migratory and invasion abilities of the cells were detected with Matrigel-uncoated and -coated Transwell inserts (8- $\mu \mathrm{m}$; EMD Millipore, Billerica, MA, USA). The detailed experiment was performed similar to previous studies $(20,21)$.

In vivo metastasis assay. Male BALB/c nude mice (4-6 weeks of age) (Centre of Laboratory Animals, The Medical College of Xi'an Jiaotong University, Xi'an, China) were randomized into two groups $(\mathrm{n}=5)$. We subsequently injected the stably overexpressing miR-577 cells, MHCC-97H-miR-577, and MHCC-97H-miR-control cells $\left(1 \times 10^{6}\right)$ into the tail veins for the establishment of a pulmonary metastatic model. Mice were sacrificed by cervical dislocation under anesthesia with ether 3 weeks post injection and examined microscopically (Axioskop 2 plus; Carl Zeiss Co., Ltd., Jena, Germany) by hematoxylin and eosin (H\&E) staining for the development of lung metastatic foci. Animals were housed in cages maintained in the pathogen-free (SPF) conditions. All in vivo protocols were approved by the Institutional Animal Care and Use Committee of Xi'an Jiaotong University. 

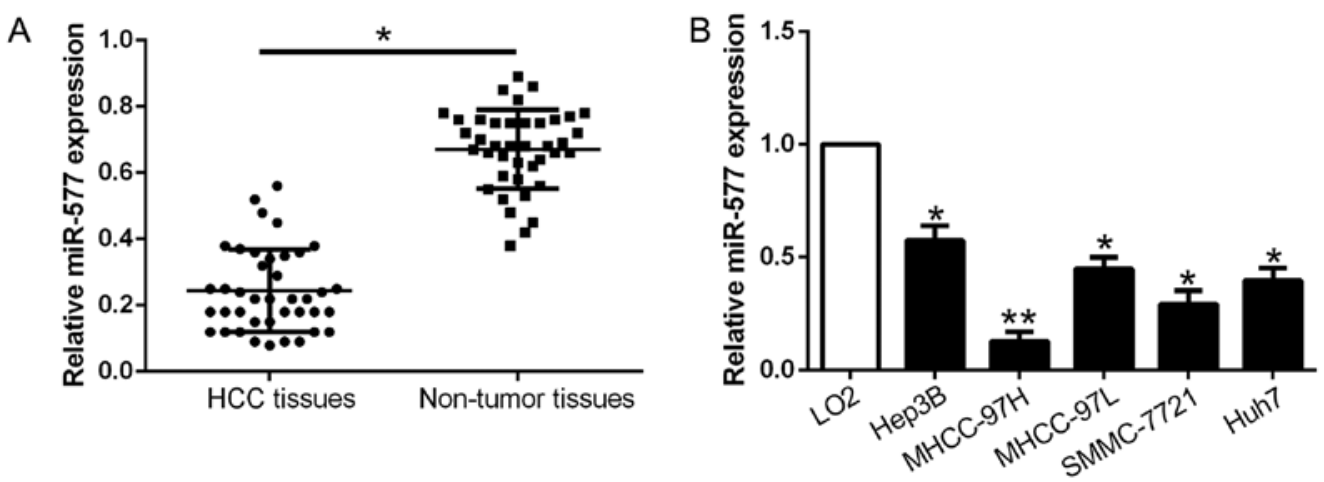

Figure 1. miR-577 expression is downregulated in HCC. (A) The expressions of miR-577 in HCC tissues was decreased when compared with that noted in the adjacent non-tumor tissues. ${ }^{*} \mathrm{P}<0.05$ vs non-tumor tissues, n=3. (B) HCC cell lines had lower expression of miR-577 than that in the normal hepatic cell line LO2. ${ }^{*} \mathrm{P}<0.05,{ }^{* *} \mathrm{P}<0.01$ vs. LO2, $\mathrm{n}=6$. HCC, hepatocellular carcinoma.

Statistical analysis. SPSS 20.0 (IBM Corp., Armonk, NY, USA) and GraphPad Prism 5.0 (GraphPad Software, Inc., La Jolla, CA, USA) were employed in this study. All data are denoted as the mean \pm SD. Statistical methods, such as one-way ANOVA, Student t-test, Kaplan-Meier method, Pearson's correlation analysis and the log-rank test were applied. A result with $\mathrm{P}<0.05$ was regarded as having a statistically significant difference.

\section{Results}

miR-577 is decreased in HCC tissues and cell lines. $q \mathrm{PCR}$ was conducted to explore miR-577 expression in 40 pairs of tumor tissues and corresponding adjacent non-tumor tissues. As exhibited in Fig. 1A, miR-577 was markedly downregulated in the HCC tissues when compared with that noted in the adjacent non-tumor tissues $(\mathrm{P}<0.05$, Fig. $1 \mathrm{~A})$. Consistently, miR-577 expression was obviously lower in the HCC cell lines compared to the normal liver cell LO2 ( $\mathrm{P}<0.05$, Fig. 1B). The above results revealed that miR-577 expression was downregulated in HCC and may play a crucial role in HCC development.

Clinical significance of miR-577 in HCC. Ninety-three patients were assigned into two subgroups (high/low miR-577 group), based on the median value of miR-577 expression in HCC tissues. We found that low miR-577 expression was notably related to venous invasion $(\mathrm{P}=0.007$, Table $\mathrm{I})$ as well as advanced tumor-node-metastasis (TNM) stage ( $\mathrm{P}=0.018$, Table I). Moreover, results from the Kaplan-Meier analysis revealed that patients with low miR-577 expression possessed worse overall survival (OS) $(\mathrm{P}=0.0001$, Fig. $2 \mathrm{~A})$ and disease-free survival (DFS) ( $\mathrm{P}=0.0001$, Fig. 2B). Thus, the above data suggest that miR-577 could be used to predict the outcome of HCC patients.

miR-577 suppresses the migration and invasion of HCC cells. miR-577 levels were manipulated by stable transfection with miR-577 mimics into MHCC-97H cells whose expression of miR-577 was the lowest, while miR-577 inhibitors were transfected into Hep3B cells which had the highest miR-577 expression $(\mathrm{P}<0.05$, Fig. 3A). MTT assays revealed that the changes in miR-577 expression did not have any significant influence on HCC cell growth compared to the control groups (Fig. 3B). Then, data from Transwell assays confirmed that overexpression of miR-577 notably suppressed the migration and invasion abilities of the MHCC-97H cells $(\mathrm{P}<0.05$, respectively, Fig. 3C), while silencing of miR-577 expression had the contrary effects on Hep3B cells $(\mathrm{P}<0.05$, respectively, Fig. 3D). To confirm the in vitro functional effects of miR-577 on HCC, we performed in vivo metastatic experiments to examine whether miR-577 could inhibit the metastasis of HCC cells in vivo. We subsequently injected the stably overexpressing miR-577 cells, MHCC-97H-miR-577 and MHCC-97H-miR-control cells into the lateral veins of the nude mice. The results showed that injection of the miR-577 overexpressing cells resulted in fewer and smaller foci in the lungs of the nude mice through microscopic evaluation (5 vs. 16 nodules per lung in MHCC-97H-miR-577 and miR-control cells, respectively; $\mathrm{P}<0.01$, Fig. 3E). Thus, we demonstrated that miR-577 exerts an anti-metastatic effect in HCC cells in vitro and in vivo.

miR-577 inhibits the EMT process of HCC cells. In order to explore the association between miR-577 and EMT, western blot analysis was performed. The results revealed that, in MHCC-97H cells, overexpression of miR-577 induced E-cadherin and suppressed $\mathrm{N}$-cadherin and vimentin $(\mathrm{P}<0.05$, Fig.4A). However, in Hep3B cells, miR-577 knockdown showed the opposite effects $(\mathrm{P}<0.05$, Fig. 4B). Moreover, we examined the metastatic phenotype of these cells and found that lung sections of the mice injected with the miR-577-overexpressing cells in fact showed increased E-cadherin expression and conversely decreased vimentin expression (Fig. 4C). Thus, our results revealed that miR-577 acts as an inhibitor of the EMT process in HCC cells.

miR-577 directly targets HOXA1 in HCC cells. Two public databases (miRanda and TargetScan) were employed to predict the potential target of miR-577 in HCC cells. Then we focused on HOXA1 and speculated HOXA1 was a candidate target, whose 3'-UTR could bind to miR-577 (Fig. 5A). Additionally, HOXA1 has been identified as an oncogene in HCC by repressing migration and invasion of HCC cells (22). To investigate whether miR-577 could interact with the 3'-UTR of HOXA1, luciferase assays were conducted. The results indicated that miR-577 negatively regulated luciferase activity of 
Table I. Correlation between the clinicopathological features and miR-577 expression in the HCC cases ( $\mathrm{n}=93)$.

\begin{tabular}{|c|c|c|c|c|}
\hline \multirow[b]{2}{*}{ Clinical parameters } & \multirow[b]{2}{*}{ Cases $(\mathrm{N})$} & \multicolumn{2}{|c|}{ Expression level } & \multirow[b]{2}{*}{ P-value } \\
\hline & & $\operatorname{miR}-577^{\text {high }}(\mathrm{n}=46)$ & $\operatorname{miR}-577^{\text {low }}(\mathrm{n}=47)$ & \\
\hline Age (years) & & & & 0.536 \\
\hline$<65$ years & 27 & 12 & 15 & \\
\hline$\geq 65$ years & 66 & 34 & 32 & \\
\hline Sex & & & & 0.838 \\
\hline Male & 74 & 37 & 37 & \\
\hline Female & 19 & 9 & 10 & \\
\hline Tumor size $(\mathrm{cm})$ & & & & 0.423 \\
\hline$<5$ & 78 & 40 & 38 & \\
\hline$\geq 5$ & 15 & 6 & 9 & \\
\hline Tumor number & & & & 0.392 \\
\hline Solitary & 80 & 41 & 39 & \\
\hline Multiple & 13 & 5 & 8 & \\
\hline Edmondson & & & & 0.166 \\
\hline $\mathrm{I}+\mathrm{II}$ & 32 & 19 & 13 & \\
\hline III+IV & 61 & 27 & 34 & \\
\hline TNM stage & & & & $0.018^{\mathrm{a}}$ \\
\hline $\mathrm{I}+\mathrm{II}$ & 76 & 42 & 34 & \\
\hline III+IV & 17 & 4 & 13 & \\
\hline Venous invasion & & & & $0.007^{\mathrm{a}}$ \\
\hline Present & 16 & 3 & 13 & \\
\hline Absent & 77 & 43 & 34 & \\
\hline $\operatorname{AFP}(\mathrm{ng} / \mathrm{ml})$ & & & & 0.667 \\
\hline$<400$ & 22 & 10 & 12 & \\
\hline$\geq 400$ & 71 & 36 & 35 & \\
\hline HBsAg & & & & 0.751 \\
\hline Positive & 84 & 42 & 42 & \\
\hline Negative & 9 & 4 & 5 & \\
\hline
\end{tabular}

${ }^{\mathrm{a}} \mathrm{P}<0.05$.
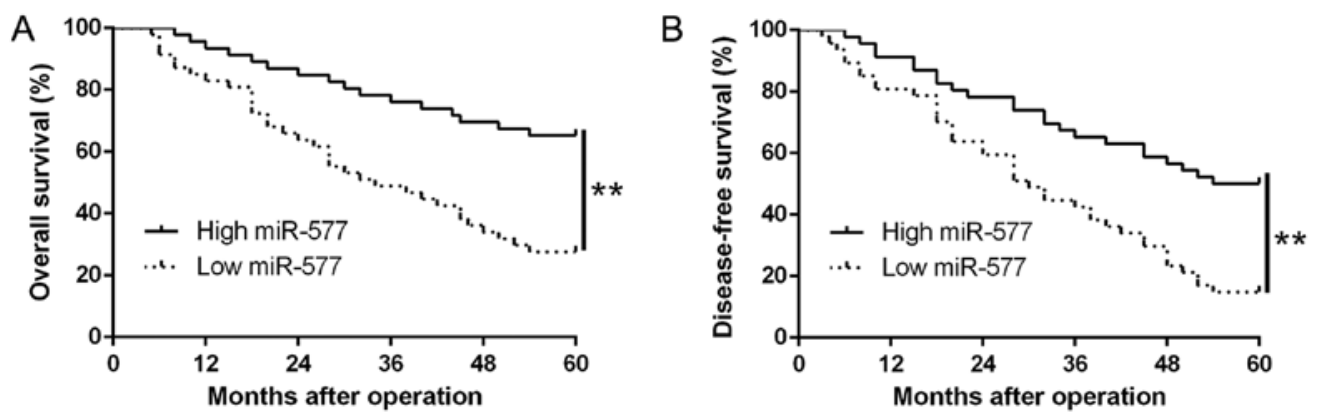

Figure 2. Clinical value of miR-577 in HCC cases. HCC patients with high miR-577 expression had a prolonged (A) 5-year overall survival (OS) and (B) disease-free survival (DFS). ${ }^{* *} \mathrm{P}<0.01$ by log-rank test vs. the miR-577 low group. HCC, hepatocellular carcinoma.

wt 3'-UTR of HOXA1 (P<0.05, Fig. 5B). However, the results could not be found in the miR-577 mutant groups (Fig. 5B). Moreover, we performed luciferase assays and found that miR-577 overexpression significantly decreased the luciferase activity of wild-type (wt) HOXA1 3'-UTR while had no influence on that of the mutant $(\mathrm{mt})$ HOXA1 3'-UTR $(\mathrm{P}<0.05$, Fig. 5B). In contrary, miR-577 knockdown increased the luciferase activity of wt HOXA1 3'-UTR (P<0.05, Fig. 5B) 
A
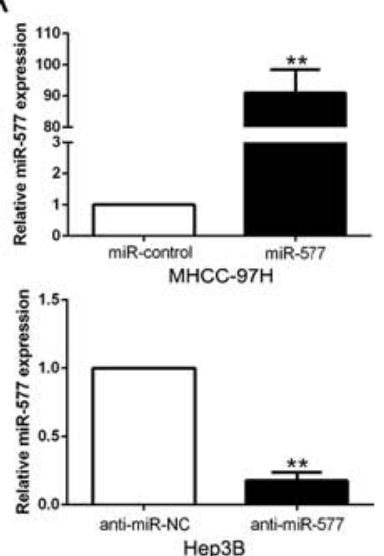

B
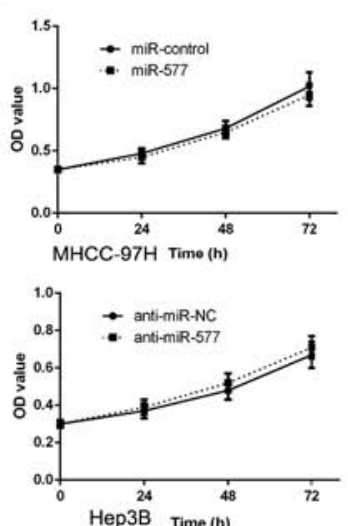

C
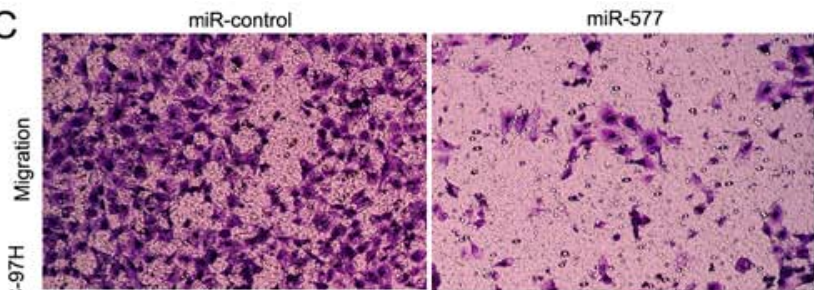

눌

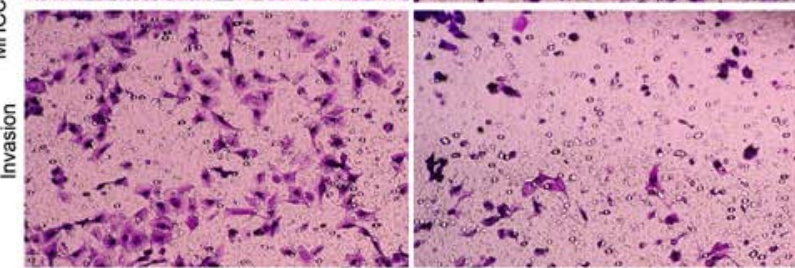

D

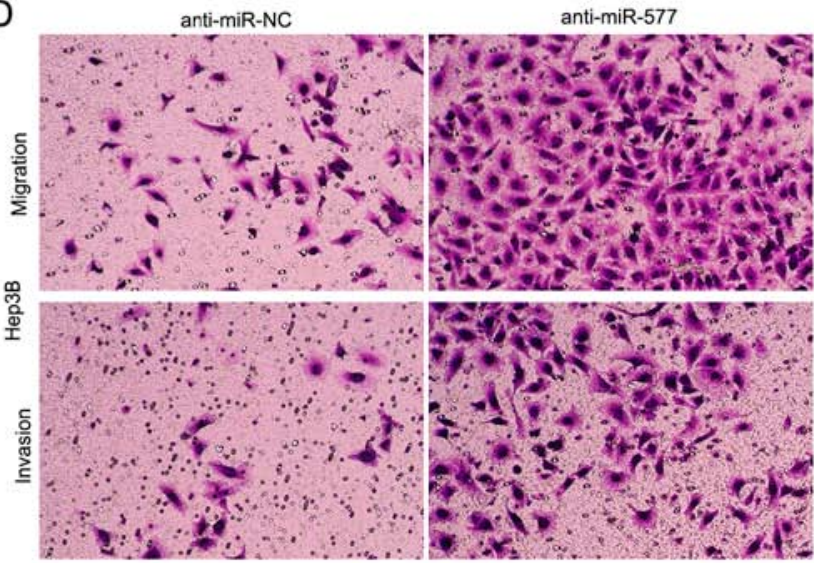

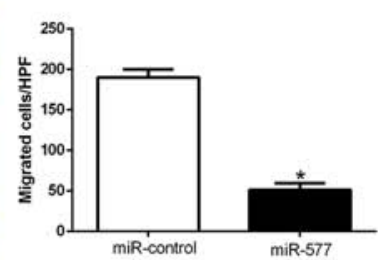

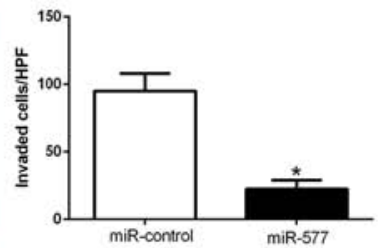

E
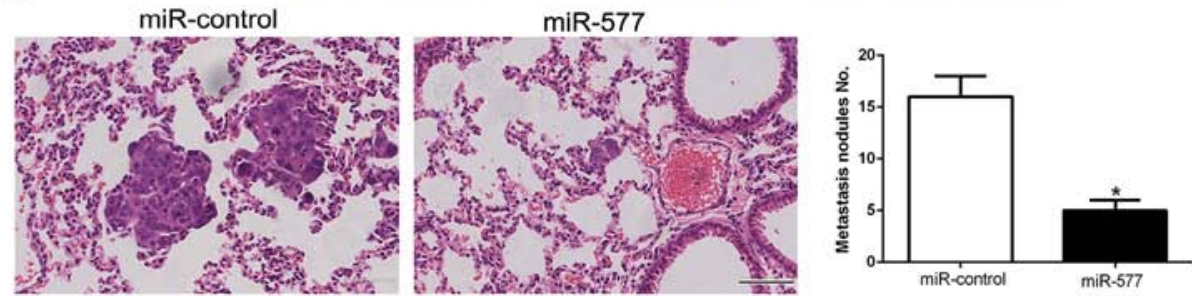

Figure 3. miR-577 is an inhibitor of HCC cell migration and invasion in vitro. (A) qPCR was performed to detect the expression of miR-577 in MHCC-97H or Hep3B cells transfected with the relevant miRNA vectors. ${ }^{* * *} \mathrm{P}<0.01$ vs. miR-control, $\mathrm{n}=6$. (B) MTT assays revealed that alteration of miR-577 had no influence on cell proliferation of HCC cells. (C) Transwell assays were employed to measure the changes in cell migration and invasion abilities in the MHCC-97H cells with overexpression of miR-577. " $\mathrm{P}<0.05$ vs. the control, $\mathrm{n}=3$. (D) Transwell assays were used to measure the changes in cell migration and invasion abilities in the Hep3B cells with miR-577 knockdown (anti-miR-577). "P<0.05 vs. the control, $n=3$. (E) Representative hematoxylin and eosin (H\&E) staining of lung metastasis of MHCC-97H-Control cells and MHCC-97H-miR-577 cells in the nude mouse pulmonary metastatic model. Magnification, x200; scale bar, $100 \mu \mathrm{m} .{ }^{*} \mathrm{P}<0.05$ vs. miR-control, $\mathrm{n}=5$. HCC, hepatocellular carcinoma; HPF, high-power field.

but did not affect the luciferase activity of mt HOXA1 3'-UTR constructs. Furthermore, results from qPCR and western blot assays revealed that both HOXA1 mRNA and protein expression were negatively regulated by miR-577 in the HCC cells $(\mathrm{P}<0.05$, respectively, Fig. 5C and D). Thus, we conclude that miR-577 directly targets HOXA1 in HCC cells.

miR-577 is inversely correlated with the expression of HOXAI in HCC tissues. Next, we attempted to determine the correlation of miR-577 and HOXA1 in HCC. The expression levels of HOXA1 mRNA and protein in HCC tissues with different miR-577 expression were detected. As expected, tissues with high miR-577 had obviously lower HOXA1 mRNA and protein expression compared to the tissues with low miR-577 $(\mathrm{P}<0.05$, Fig. 6A and B). Furthermore, there existed a negative correlation between HOXA1 mRNA and miR-577 in the HCC tissues $\left(\mathrm{R}^{2}=0.6866, \mathrm{P}<0.001\right.$, Fig. $\left.6 \mathrm{C}\right)$. In addition, we performed western blot analysis to confirm that HOXA1 was overexpressed in HCC tissues compared to that in the corresponding adjacent non-tumor tissues ( $\mathrm{P}<0.05$, Fig. 6D). These results confirmed that HOXA1 acts as a downstream target of miR-577 in HCC, and HOXA1 is negatively regulated by miR-577 in HCC.

Restoration of HOXA1 reverses the biological effects of miR-577 on HCC cells. To determine whether HOXA1 abrogates the effects of miR-577, we restored HOXA1 expression in miR-577-overexpressing MHCC-97H cells ( $\mathrm{P}<0.05$, Fig. 7A). Interestingly, regaining HOXA1 partially abrogated the inhibitory functions of the ovexpression of miR-577 in regards to migration, invasion and EMT of MHCC-97H cells $(\mathrm{P}<0.05$, Fig. 7B and E). In contrast, HOXA1 inhibition by a specific 

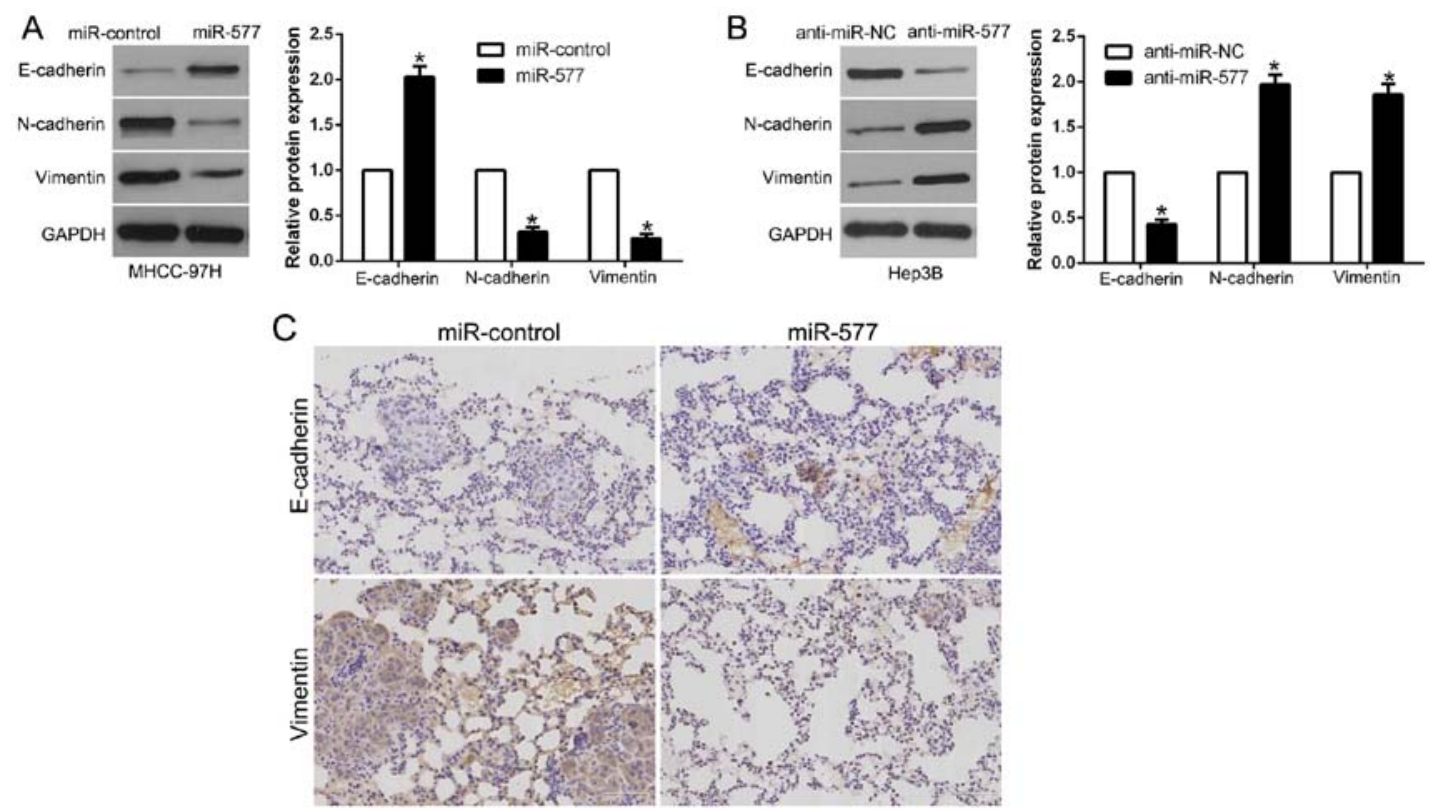

Figure 4. miR-577 suppresses the process of EMT in HCC cells. (A) Expression of epithelial cell marker (E-cadherin) was increased, while the expression of mesenchymal cell markers (N-cadherin and vimentin) were decreased following overexpression of miR-577 in the MHCC-97H cells. (B) In contrast, miR-577 knockdown downregulated E-cadherin expression and upregulated the expression of $\mathrm{N}$-cadherin and vimentin in the Hep3B cells. ${ }^{*} \mathrm{P}<0.05$ vs. the control, $\mathrm{n}=3$. (C) Representative images of immunochemical staining of serial lung sections for E-cadherin and vimentin staining in the MHCC-97H-control (miR-control) group and the MHCC-97H-miR-577 (miR-577) group. EMT, epithelial-to-mesenchymal transition; HCC, hepatocellular carcinoma.

A

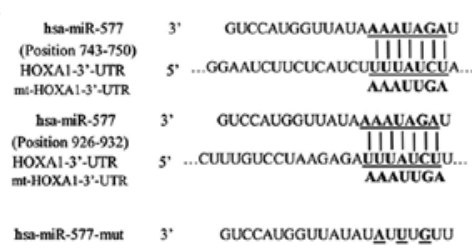

C
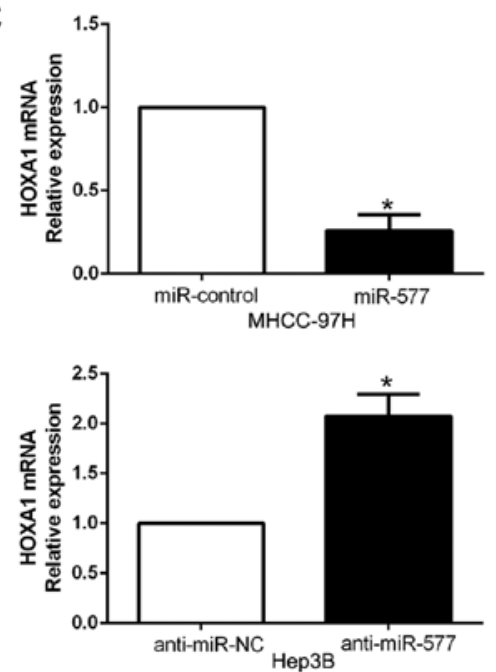

B
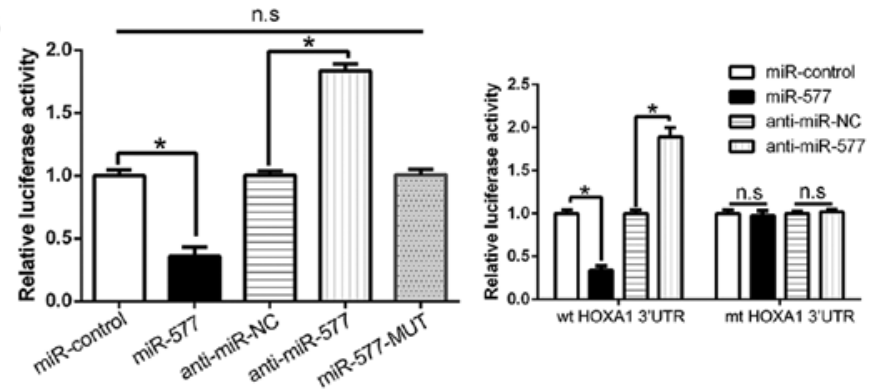

D
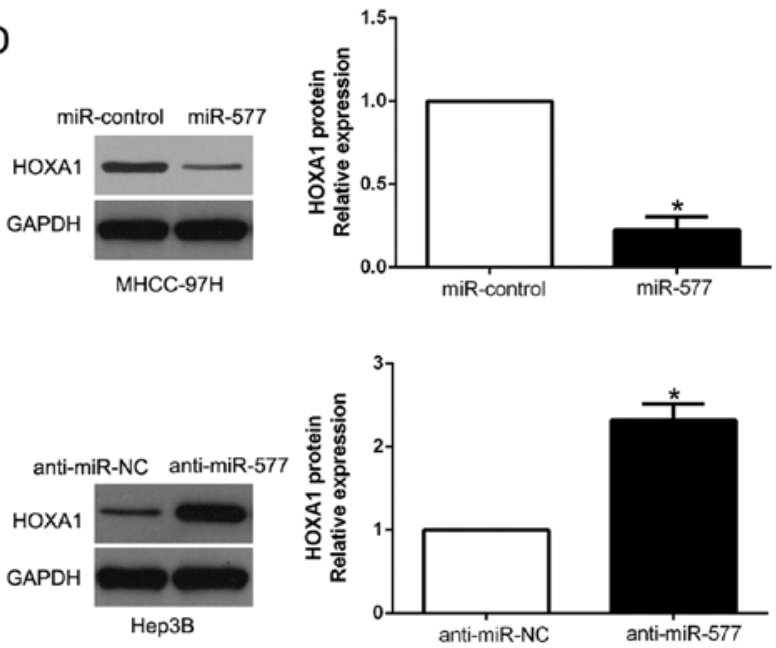

Figure 5. HOXA1 is identified to be a direct downstream target of miR-577 in HCC. (A) Bioinformatic analysis revealed that there exists a putative binding sequence for miR-577 in the 3'-UTR of HOXA1. Mutant miR-577 with mutated seed region complementary to the 3'-UTR of HOXA1 was constructed. (B) Overexpression of wild-type (wt) miR-577 markedly reduced the luciferase activity of HOXA1 3'-UTR. In contrast, repression of wild-type miR-577 revealed the contrary influences. Alteration of mutant (mt) miR-577 did not have notable effects on the luciferase activity of HOXA1 3'-UTR. "P<0.05 vs. the control, $n=3$. miR-577 significantly suppressed the luciferase activity that carried the wild-type but not mutant 3'-UTR of HOXA1. Anti-miR-577 led to a notable increase in the luciferase activity of wt 3'-UTR of HOXA1. "P<0.05 vs. the control, $n=3$. (C) The mRNA expression levels of HOXA1 in MHCC-97H cells transfected with the miR-control or miR-577 mimics and Hep3B cells transfected with anti-miR-NC and miR-577 inhibitors were measured by qPCR. ${ }^{*} \mathrm{P}<0.05$ vs. the control, $n=3$. (D) The expression of HOXA1 protein in MHCC-97H cells was reduced by overexpression of miR-577 and knockdown of miR-577 of Hep3B cells enhanced the expression level of HOXA1 protein. ${ }^{\text {P }}<0.05$ vs. the control; n.s., not significant; $n=3$. HCC, hepatocellular carcinoma; HOXA1, homeobox A1. 

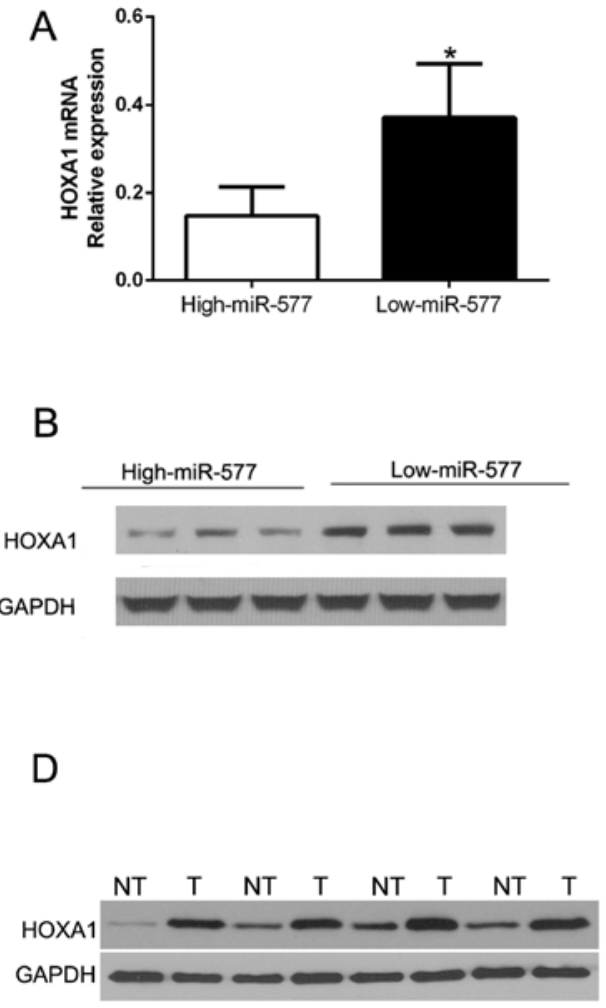
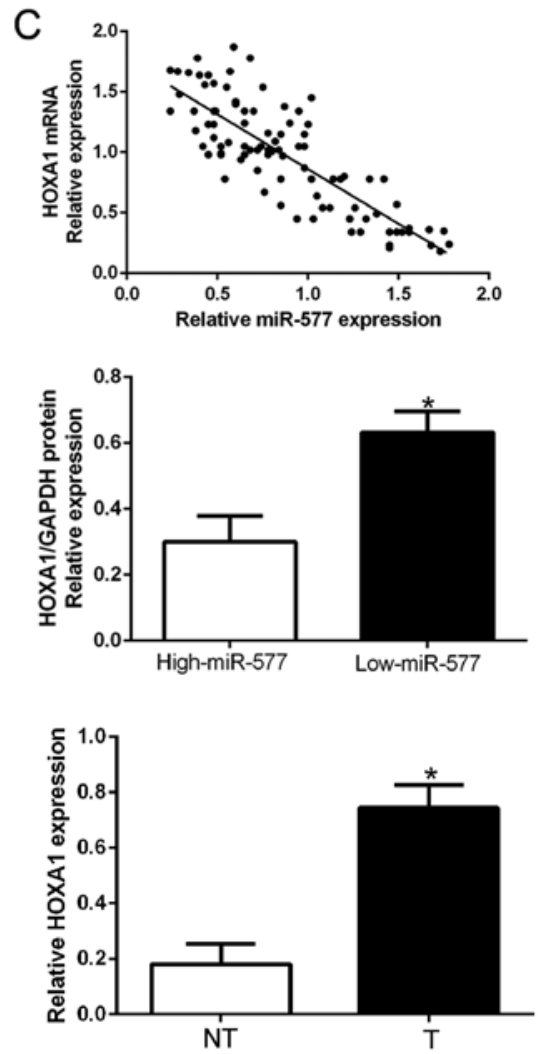

Figure 6. miR-577 negatively regulates HOXA1 expression in HCC cells. The expression of HOXA1 mRNA (A) and protein (B) in tumor tissues of the high miR-577 group was markedly lower than that in tumor tissues of the low miR-577 group. $\mathrm{N}$ (high-miR-577)=46, $\mathrm{N}$ (low-miR-577)=47. ${ }^{*} \mathrm{P}<0.05 \mathrm{vs}$. the miR-577-low group. (C) The mRNA level of HOXA1 was found to be negatively correlated $\left(\mathrm{R}^{2}=0.6866\right)$ with miR-577 expression in HCC tissues. (D) The HOXA1 expression in HCC tissues (T) was overexpressed compared to that noted in the adjacent non-tumor tissues (NT). " $\mathrm{P}<0.05$ vs. NT. HCC, hepatocellular carcinoma; HOXA1, homeobox A1.

siRNA significantly reversed the promotive effects of miR-577 knockdown in Hep3B cells $(\mathrm{P}<0.05$, Fig. $7 \mathrm{C}, \mathrm{D}$ and $\mathrm{F})$. In brief, our findings demonstrated that HOXA1 reversed the anti-metastatic effects of miR-577 in HCC cells.

\section{Discussion}

Cumulative evidence indicates that miRNAs take part in the progression of human malignancies as either oncogenes or tumor suppressors (23). Recently, miR-577 was identified as a new tumor-related miRNA. In esophageal squamous cell carcinoma, miR-577 was found to regulate cell proliferation and the cell cycle by targeting TSGA10 (24). Moreover, miR-577 was found to be involved in non-alcoholic fatty liver disease (25). In the present study, both in HCC tissues and cell lines, miR-577 was notably underexpressed. Downregulation of miR-577 was closely related to poor clinicopathological characteristics of HCC patients. Importantly, we demonstrated that HCC patients in the low miR-577 group exhibited an obviously worse 5-year overall survival and disease-free survival. These results indicate an important role of miR-577 in HCC development and could be a predictor of patient survival for HCC patients. Therefore, these data suggest that reduced miR-577 might be able to serve as a potential biomarker for $\mathrm{HCC}$, and a prognostic indicator for HCC patients.

Metastasis is one of the main causes of treatment failure and poor outcome in HCC patients. During the initiation of metastasis, EMT is a vital step. In this research, by gain- and loss-of-function experiments, miR-577 was identified to be an inhibitor of HCC cell migration and invasion in vitro and in vivo. The same biological effects of miR-577 were also noted in other cancers $(10,11)$. Nevertheless, alteration of miR-577 had no effect on HCC cell proliferation. Moreover, miR-577 suppressed HCC cell EMT process. Moreover, in lung metastatic tissues, miR-577 overexpression also inhibited the EMT process. Thus, our findings indicated that miR-577 suppresses HCC metastasis via influencing EMT.

HOXA1, which is a member of the HOX gene family, regulates cell differentiation, embryonic development, survival and migration. Increasing evidence has confirmed that HOXA1 expression is dysregulated in diverse cancer types (26-29). In small cell lung cancer, HOXA1 is targeted by miR-100 to regulate tumor cell growth and chemoresistance (30). In gastric cancer, elevated HOXA1 acts as an oncogene to promote tumor cell proliferation (31). In HCC, overexpression of HOXA1 promotes cell growth, migration and invasion and is closely related to the poor prognosis of HCC patients (22). We also confirmed that HOXA1 is overexpressed in HCC compared to that noted in corresponding adjacent non-tumor tissues, which shows a similar result. These data confirmed the critical roles of HOXA1 in cancer development. Here, our data revealed that miR-577 modulated HCC cell metastasis and the process of EMT by directly interacting with HOXA1. However, how HOXA1 regulates the EMT process remains unclear. miR-577 was found to negatively regulate HOXA1 expression in HCC cells and to change the luciferase activity 
A
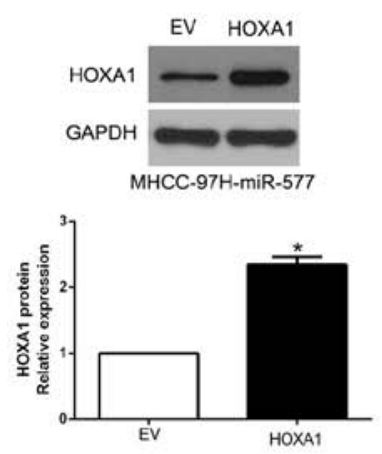

C
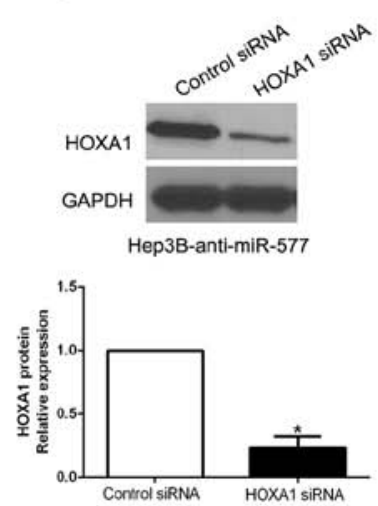

E

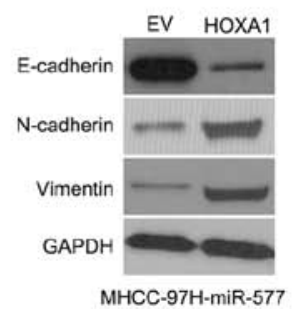

B

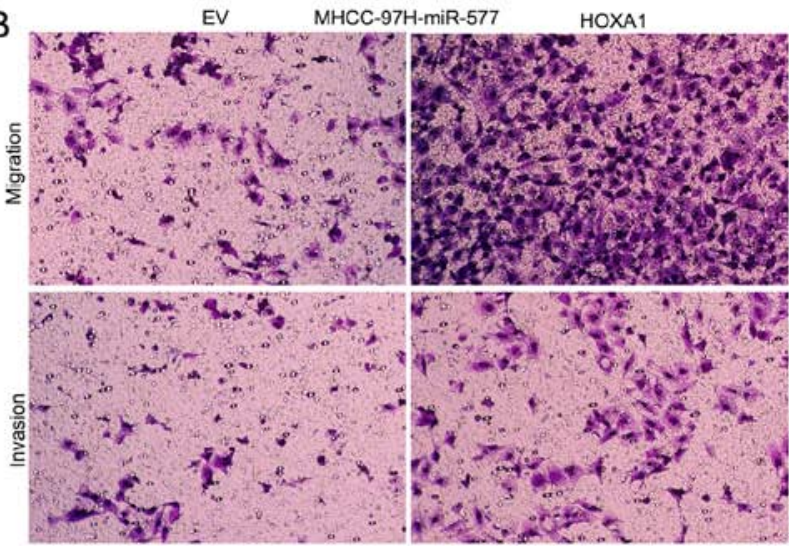

D

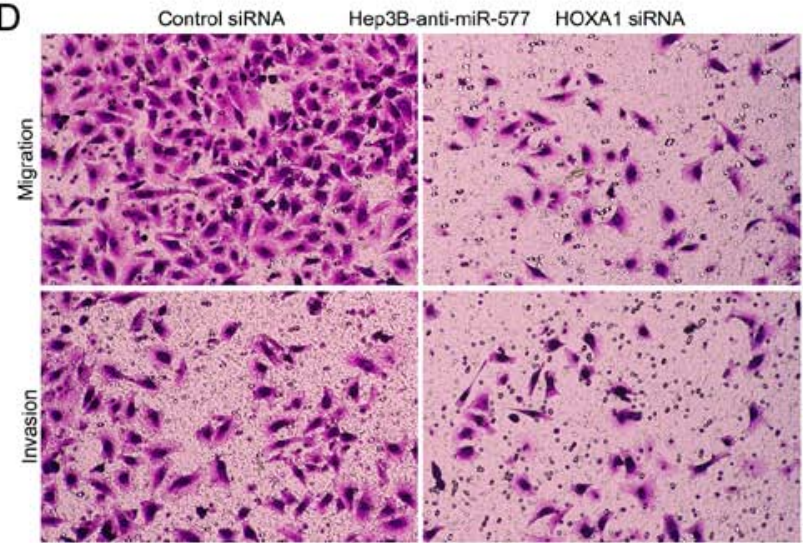

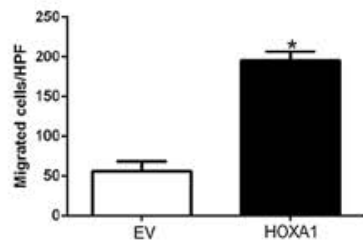

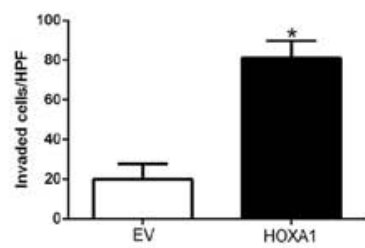

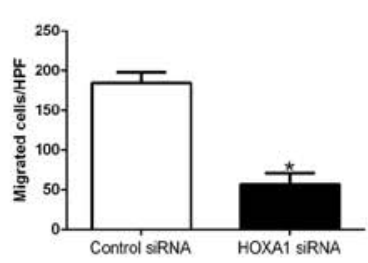

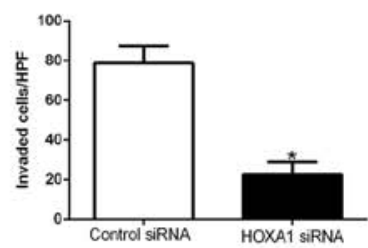

$\mathrm{F}$

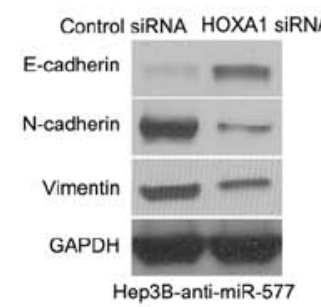

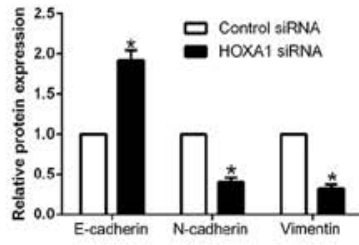

Figure 7. HOXA1 reverses the effects of miR-577 on HCC cell migration, invasion and EMT. (A) Western blot analysis was employed to explore the expression of HOXA1 protein in miR-577-overexpressing MHCC- $97 \mathrm{H}$ cells which were simultaneously transfected with EV or HOXA1 clones. ${ }^{*} \mathrm{P}<0.05$ vs. EV, $\mathrm{n}=3$. (B) Upregulation of HOXA1 abolished the effects on cell migration and invasion in the miR-577-overexpressing MHCC-97H cells. "P<0.05 vs. EV, $\mathrm{n}=3$. (C) Western blot analysis was used for the detection of HOXA1 protein in miR-577-silenced Hep3B cells which were transfected with control siRNA or HOXA1 siRNA. "P<0.05 vs. HOXA1 siRNA, n=3. (D) Knockdown of HOXA1 reversed the effects of anti-miR-577 on cell migration and invasion in the Hep3B cells. "P<0.05 vs. HOXA1 siRNA, n=3. (E) Western blot analysis was used to detect the expression of EMT markers in MHCC-97H cells co-transfected with miR-577 mimics and HOXA1 or control vector. (F) The expression of EMT markers in Hep3B cells co-transfected with miR-577 inhibitor and HOXA1 siRNA or control siRNA were detected with western blot analysis. EV, empty vector. ${ }^{*} \mathrm{P}<0.05$ vs. the control, $\mathrm{n}=3$. HPF, high-power field.

of 3'-UTR of HOXA1-wt, rather than 3'-UTR of HOXA1-mt. Moreover, restoration of HOXA1 significantly reversed the effect of miR-577 on HCC cell migration, invasion and EMT. However, the detailed molecular mechanism of HOXA1 downstream pathway warrants further investigation. In a word, HOXA1, a downstream target of miR-577, reversed the inhibitory effects of miR-577 on HCC cell migration, invasion and EMT process.

In conclusion, aberrant expression of microRNAs (miRNAs) is closely associated with HCC pathogenesis and tumorigenicity. Recent studies suggest that miR-577 is a cancer-related miRNA. In the present study, both in HCC tissues and cell lines, miR-577 expression was found to be downregulated. Decreased miR-577 was distinctly related to malignant clinicopathologic features and worse outcome of HCC patients. Functionally, miR-577 modulated HCC cell migration, invasion and EMT. Additionally, miR-577 directly targets HOXA1 to exert its effects on HCC cells. Taken together, miR-577 could act as a prognostic tumor biomarker and a potential target for molecular-targeted therapy of HCC.

\section{Acknowledgements}

Not applicable.

\section{Funding}

This study was supported by grants from the National Natural Science Foundation of China (nos. 81602566, 81402039 and 
81572847), and the Natural Science Basic Research Plan in Shaanxi Province of China (no. 2016JQ8029).

\section{Availability of data and materials}

The datasets used during the present study are available from the corresponding author upon reasonable request.

\section{Authors' contributions}

QGL and SSH conceived and designed the experiments; ZKL, YFW, LW, BWY, CG and TS performed the experiments; SSH and ZKL analyzed the data; KST and SSH contributed reagents/materials/analysis tools; ZKL and $\mathrm{SSH}$ wrote the paper. All authors read and approved the manuscript and agree to be accountable for all aspects of the research in ensuring that the accuracy or integrity of any part of the work are appropriately investigated and resolved.

\section{Ethics approval and consent to participate}

All of the patients provided written informed consent. Xi'an Jiaotong University Ethics Committee (Xi'an, China) approved the research on the basis of the Declaration of Helsinki.

\section{Consent for publication}

Not applicable.

\section{Competing interests}

The authors declare that they have no competing interests.

\section{References}

1. Torre LA, Bray F, Siegel RL, Ferlay J, Lortet-Tieulent J and Jemal A: Global cancer statistics, 2012. CA Cancer J Clin 65 87-108, 2015.

2. El-Serag HB and Rudolph KL: Hepatocellular carcinoma: Epidemiology and molecular carcinogenesis. Gastroenterology 132: 2557-2576, 2007.

3. Forner A, Llovet JM and Bruix J: Hepatocellular carcinoma. Lancet 379: 1245-1255, 2012.

4. Maluccio M and Covey A: Recent progress in understanding, diagnosing, and treating hepatocellular carcinoma. CA Cancer J Clin 62: 394-399, 2012.

5. Garzon R, Calin GA and Croce CM: MicroRNAs in cancer. Ann Rev Med 60: 167-179, 2009.

6. Bartel DP: MicroRNAs: Genomics, biogenesis, mechanism, and function. Cell 116: 281-297, 2004.

7. Tu K, Liu Z, Yao B, Han S and Yang W: MicroRNA-519a promotes tumor growth by targeting PTEN/PI3K/AKT signaling in hepatocellular carcinoma. Int J Oncol 48: 965-974, 2016.

8. Ji H, Chen M, Greening DW, He W, Rai A, Zhang W and Simpson RJ: Deep sequencing of RNA from three different extracellular vesicle (EV) subtypes released from the human LIM1863 colon cancer cell line uncovers distinct miRNA-enrichment signatures. PloS One 9: e110314, 2014.

9. Wang LY, Li B, Jiang HH, Zhuang LW and Liu Y: Inhibition effect of miR-577 on hepatocellular carcinoma cell growth via targeting $\beta$-catenin. Asian Pac J Trop Med 8: 923-929, 2015.

10. Zhang W, Shen C, Li C, Yang G, Liu H, Chen X, Zhu D, Zou H, Zhen Y, Zhang D and Zhao S: miR-577 inhibits glioblastoma tumor growth via the Wnt signaling pathway. Mol Carcinog 55: $575-585,2016$.
11. Yu Z, Zhang W and Deng F: MicroRNA-577 inhibits gastric cancer growth by targeting E2F transcription factor 3. Oncol Lett 10: 1447-1452, 2015.

12. Jiang H, Ju H, Zhang L, Lu H and Jie K: microRNA-577 suppresses tumor growth and enhances chemosensitivity in colorectal cancer. J Biochem Mol Toxicol 31, 2017.

13. Chen XY, Li GM, Dong Q and Peng H: MiR-577 inhibits pancreatic $\beta$-cell function and survival by targeting fibroblast growth factor 21 (FGF-21) in pediatric diabetes. Genet Mol Res 14: 15462-15470, 2015.

14. Yamanaka C, Wada H, Eguchi H, Hatano H, Gotoh K, Noda T, Yamada D, Asaoka T, Kawamoto K, Nagano H, et al: Clinical significance of CD13 and epithelial mesenchymal transition (EMT) markers in hepatocellular carcinoma. Jpn J Clin Oncol 48: 52-60, 2018.

15. Vincent CT and Fuxe J: EMT, inflammation and metastasis. Semin Cancer Biol 47: 168-169, 2017.

16. Lamouille S, Xu J and Derynck R: Molecular mechanisms of epithelial-mesenchymal transition. Nat Rev Mol Cell Biol 15: 178-196, 2014.

17. Guarino M, Rubino B and Ballabio G: The role of epithelial-mesenchymal transition in cancer pathology. Pathology 39: 305-318, 2007.

18. Liu Z, Dou C, Yao B, Xu M, Ding L, Wang Y, Jia Y, Li Q, Zhang $\mathrm{H}$, Tu K, et al: Ftx non coding RNA-derived miR-545 promotes cell proliferation by targeting RIG-I in hepatocellular carcinoma. Oncotarget 7: 25350-25365, 2016.

19. Liu Z, Dou C, Jia Y, Li Q, Zheng X, Yao Y, Liu Q and Song T: RIG-I suppresses the migration and invasion of hepatocellular carcinoma cells by regulating MMP9. Int J Oncol 46: 1710-1720, 2015.

20. Liu Z, Dou C, Yao B, Xu M, Ding L, Wang Y, Jia Y, Li Q, Zhang $\mathrm{H}$, Tu K, et al: Methylation-mediated repression of microRNA-129-2 suppresses cell aggressiveness by inhibiting high mobility group box 1 in human hepatocellular carcinoma. Oncotarget 7: 36909-36923, 2016.

21. Liu Z, Dou C, Wang Y, Jia Y, Li Q, Zheng X, Yao Y, Liu Q and Song T: High-mobility group box 1 has a prognostic role and contributes to epithelial mesenchymal transition in human hepatocellular carcinoma. Mol Med Rep 12: 5997-6004, 2015.

22. Zha TZ, Hu BS, Yu HF, Tan YF, Zhang Y and Zhang K: Overexpression of HOXA1 correlates with poor prognosis in patients with hepatocellular carcinoma. Tumour Biol 33: 2125-2134, 2012.

23. Dou C, Liu Z, Xu M, Jia Y, Wang Y, Li Q, Yang W, Zheng X, Tu K and Liu Q: miR-187-3p inhibits the metastasis and epithelial-mesenchymal transition of hepatocellular carcinoma by targeting S100A4. Cancer Lett 381: 380-390, 2016.

24. Yuan X, He J, Sun F and Gu J: Effects and interactions of MiR-577 and TSGA10 in regulating esophageal squamous cell carcinoma. Int J Clin Exp Pathol 6: 2651-2667, 2013.

25. Li Z, Feng S, Zhou L, Liu S and Cheng J: NS5ATP6 modulates intracellular triglyceride content through FGF21 and independently of SIRT1 and SREBP1. Biochem Biophys Res Commun 475: 133-139, 2016.

26. Li Q, Zhang X, Li N, Liu Q and Chen D: miR-30b inhibits cancer cell growth, migration, and invasion by targeting homeobox A1 in esophageal cancer. Biochem Biophys Res Commun 485: 506-512, 2017.

27. Wang H, Liu G, Shen D, Ye H, Huang J, Jiao L and Sun Y: HOXA1 enhances the cell proliferation, invasion and metastasis of prostate cancer cells. Oncol Rep 34: 1203-1210, 2015.

28. Wang X, Li Y, Qi W, Zhang N, Sun M, Huo Q, Cai C, Lv S and Yang Q: MicroRNA-99a inhibits tumor aggressive phenotypes through regulating HOXA1 in breast cancer cells. Oncotarget 6: 32737-32747, 2015.

29. Kraft S, Moore JB, Muzikansky A, Scott KL and Duncan LM: Differential UBE2C and HOXA1 expression in melanocytic nevi and melanoma. J Cutan Pathol 44: 843-850, 2017.

30. Xiao F, Bai Y, Chen Z, Li Y, Luo L, Huang J, Yang J, Liao $\mathrm{H}$ and Guo L: Downregulation of HOXA1 gene affects small cell lung cancer cell survival and chemoresistance under the regulation of miR-100. Eur J Cancer 50: 1541-1554, 2014.

31. Yuan C, Zhu X, Han Y, Song C, Liu C, Lu S, Zhang M, Yu F, Peng $\mathrm{Z}$ and Zhou C: Elevated HOXA1 expression correlates with accelerated tumor cell proliferation and poor prognosis in gastric cancer partly via cyclin D1. J Exp Clin Cancer Res 35: 15, 2016. 